\title{
Eclecticism, Diversity and the Practice of Educational and Developmental Psychology - ERRATUM
}

doi: 10.1017/edp.2013.21, published online by Cambridge University Press January 2014

The above editorial has been updated to include the name of the editor as the author. The publishers would like to apologise for this error.

Chris Boyle PhD

Editor

\section{Reference}

Boyle, C. (2013). Eclecticism, Diversity and the Practice of Educational and Developmental Psychology. The Australian Educational and Developmental Psychologist, 30, pp iii-iv. doi:10.1017/edp.2013.21. 